\title{
Interfacial effects in manganite thin films with different capping layers of interest for spintronic applications
}

\author{
S. Valencia, ${ }^{1}$ Z. Konstantinovic, ${ }^{2}$ D. Schmitz, ${ }^{1}$ A. Gaupp, ${ }^{1}$ Ll. Balcells, ${ }^{2}$ and B. Martínez ${ }^{2}$ \\ ${ }^{1}$ Helmholtz-Zentrum-Berlin für Materialien und Energie, Albert-Einstein Str. 15 D-12489 Berlin, Germany \\ ${ }^{2}$ Instituto de Ciencia de Materiales de Barcelona-CSIC, Campus UAB. E-08193 Bellaterra, Spain
}

(Received 22 February 2011; revised manuscript received 3 May 2011; published 8 July 2011)

\begin{abstract}
We report on the use of surface-sensitive techniques (x-ray absorption spectroscopy and $\mathrm{x}$-ray magnetic circular dichroism) to investigate interfacial effects in sputtered manganite thin films, $\mathrm{La}_{2 / 3} \mathrm{Ca}_{1 / 3} \mathrm{MnO}_{3}$ (LCMO) and $\mathrm{La}_{2 / 3} \mathrm{Sr}_{1 / 3} \mathrm{MnO}_{3}$ (LSMO), with different capping layers [MgO, $\mathrm{LaAlO}_{3}$ (LAO), $\mathrm{SrTiO}_{3}(\mathrm{STO}), \mathrm{NdGaO}_{3}$ (NGO), and $\mathrm{Au}$. To ensure surface sensitivity, data were acquired by using the total electron yield detection mode. It is found that LSMO and LCMO films exhibit similar behavior when capped with oxide layers but dissimilar effects when a metallic capping is used. Almost bulklike Mn valence at the interface was observed in the case of LAO capping. However, a notorious increase of the Mn oxidation state was detected for both $\mathrm{MgO}$ and NGO capping layers. In contrast, metallic Au and STO capping promotes a reduction of the Mn oxidation state. These results are correlated with the x-ray magnetic circular dichroism data, showing a concomitant decrease of the saturation magnetization at the interface in those cases where a modification of the Mn oxidation state is observed.
\end{abstract}

DOI: 10.1103/PhysRevB.84.024413

PACS number(s): 78.70.Dm, 75.47.Lx, 75.70.-i, 71.20.-b

\section{INTRODUCTION}

Heterointerfaces between complex oxides with perovskite structure have evidenced a variety of unexpected properties going from the formation of a two-dimensional (2D) electron gas at the interface between two insulators to the appearance of interfacial ferromagnetism adjoining two nonmagnetic oxides. ${ }^{1-5}$ Those results make evident that the magnetic and electronic properties of oxide-based heterostructures can be tuned through interfacial effects, such as strain, charge transfer, and spin exchange interactions, offering the possibility of exploiting new functionalities for the design of new oxidebased devices. In this context, most of the efforts have concentrated on the study of the high-mobility metallic state appearing in heterostructures combining two band insulators. ${ }^{1}$

For obvious reasons, interfacial effects are very important in the case of tunneling devices, such as magnetic tunneling junctions, in which the tunneling current depends critically on the magnetic and electronic properties of the electrode-barrier interface. This fact has motivated intensive investigations of interfacial effects in manganite thin films with different complex oxides. Manganese perovskites are complex systems displaying a broad range of physical phenomena, including large spin polarization, colossal magnetoresistance, electronic phase segregation, orbital ordering, charge ordering, etc., ${ }^{6}$ rendering them as the ideal materials to develop novel concepts of oxideelectronic devices. Due to these very promising technological perspectives, strong activity is maintained in the preparation of manganite thin films, especially in $\mathrm{La}_{1-x} \mathrm{~A}_{x} \mathrm{MnO}_{3}(\mathrm{~A}=$ $\mathrm{Sr}, \mathrm{Ca})$ systems at the optimal doping rate $x=1 / 3$ that corresponds to the highest magnetic transition temperature. ${ }^{7}$ The performances of magnetic tunneling devices depend critically on the conducting properties of the few atomic layers next to the insulating barrier. In the case of manganites, several causes, such as strain, structural defects, surface roughness, oxygen stoichiometry, and doping level, might contribute to depress the magnetic and transport properties at the interface.
Among $\mathrm{La}_{1-x} \mathrm{~A}_{x} \mathrm{MnO}_{3}$ perovskites $\mathrm{La}_{2 / 3} \mathrm{Sr}_{1 / 3} \mathrm{MnO}_{3}$ (LSMO) is the most interesting one from a technological point of view, due to its high Curie temperature $\left(T_{C} \sim\right.$ $370 \mathrm{~K})$. In this context, the most studied oxide interface system is the $\mathrm{LSMO} \mathrm{SrTiO}_{3}$ (STO) interface. ${ }^{3-10}$ Interfaces with other oxides of interest, such as $\mathrm{LaAlO}_{3}$ (LAO) or $\mathrm{MgO}$, have been less investigated. ${ }^{11-13}$ Several studies conducted to analyze the properties of ultrathin manganite films on top of various substrates indicate a severe degradation of the magnetotransport properties below a critical thickness. ${ }^{14,15}$ The origin of this degradation has usually been attributed to the existence of phase segregation and disorder at the nanoscale motivated mainly by structural strain, oxygen stoichiometry, and variations of the chemical composition. ${ }^{6,16}$

To gain a deeper insight into the mechanisms behind the interfacial degradation of the magnetotransport properties, the use of interface-selective probing techniques is required. In this sense, some studies have already been conducted. For instance, in Ref. 3, magnetization-induced second-harmonic generation was used to locally probe the magnetic properties at the interface of LSMO with nonmagnetic insulating layers, showing that STO produces a magnetic dead layer in the adjacent LSMO more aggressively than LAO does because the STO layer acts as a hole-donating layer. On the other hand, high-resolution transmission electron microscopy (HRTEM) jointly with electron energy loss spectroscopy (EELS) has also been used to investigate manganite-oxide interfaces ${ }^{9,10,17}$ aimed to determine not only the chemical composition of the interface areas, but also the effect of the neighboring atoms on their electronic structure. Nevertheless, the role of each of those parameters, namely structural strain, oxygen stoichiometry, and variations of the chemical composition, and how they interact with each other is not well understood yet. One of the reasons is that most of the literature reporting on interfacial effects is based on the growth of manganite films on top of different substrates, presenting thus different strain conditions. 
In this context, the use of surface, element-, and magneticsensitive $\mathrm{x}$-ray spectroscopy techniques are very helpful to clarify the microscopic origin of the depression of the magnetotransport properties at the interfaces. ${ }^{18-20}$ In this work, we report on interfacial effects in LSMO and $\mathrm{La}_{2 / 3} \mathrm{Ca}_{1 / 3} \mathrm{MnO}_{3}$ (LCMO) manganite thin films prepared by sputtering with different capping layers $\left[\mathrm{MgO}, \mathrm{LAO}, \mathrm{STO}, \mathrm{NdGaO}_{3}(\mathrm{NGO})\right.$ and gold]. All films were grown on top of STO (001)-oriented substrates under the very same deposition conditions. This guarantees that both LCMO and LSMO films present the same strain conditions independently of the capping, allowing therefore a direct look into film-capping layer chemical modifications. We have investigated possible changes of both the chemical composition and magnetic properties at the interface area. With this aim, we have measured the x-ray absorption spectra (XAS) and the x-ray magnetic circular dichroism (XMCD) at various fields. Data were acquired by using the total electron yield (TEY) detection mode, thus guaranteeing interface sensitivity. The results show that LAO capping does not modify the bulklike $\mathrm{Mn}$ valence at the interface in a significant way. In contrast, $\mathrm{MgO}$ and $\mathrm{NGO}$ capping lead to an increase of the Mn oxidation state for both LSMO and LCMO thin films. Au and STO induce divalent Mn formation on LSMO only. These results are correlated with the XMCD data, showing a concomitant decrease of the saturation magnetization of those interfaces in which $\mathrm{Mn}^{3+} / \mathrm{Mn}^{4+}$ valency balance corresponding to the nominal 2/3-1/3 composition is modified.

\section{EXPERIMENTAL}

Manganite films were grown on (001)-oriented STO substrates by using radio frequency (RF) magnetron sputtering from stoichiometric ceramic targets. Prior to deposition, substrates were cleaned in an ultrasonic bath with Milli-Q water and annealed at $1000{ }^{\circ} \mathrm{C}$ in air for $2 \mathrm{~h}$ to obtain a typical morphology of terraces and steps with unit cell height $(\sim 0.4 \mathrm{~nm})$. Further details regarding sample preparation can be found elsewhere. ${ }^{21}$ The thickness of the samples $(\sim 50 \mathrm{~nm})$ was determined from grazing incident $\mathrm{x}$-ray reflectometry. The thickness of the capping layers was set to $1.6( \pm 0.2)$ $\mathrm{nm}$ and was determined by controlling the evaporation time after a careful calibration of the growth rate of each of the different materials used. Reciprocal space mapping was performed using a Bruker D8 GADDS system equipped with a $2 \mathrm{D}$ Hi-Star $\mathrm{x}$-ray detector to determine the degree of strain of the films. The surface morphology was analyzed by atomic force microscopy (AFM) working in the tapping mode. Macroscopic magnetic characterization of the samples was performed by using a commercial SQUID magnetometer (Quantum Design). Synchrotron experiments were performed at the electron storage ring BESSY II by using the 7 Tesla high-field end station located at the UE46-PGM1 beamline. The measurements were done at $T=10 \mathrm{~K}$, i.e. well within the ferromagnetic (FM) phase of the perovskite compounds. The incoming circularly polarized radiation impinged the sample at normal incidence with respect to its surface plane. XAS and XMCD spectra at the Mn $L_{3,2}$-edge were obtained at various fields ranging from 0 to $6 \mathrm{~T}$ applied perpendicular to sample surface. Within this configuration, magnetic saturation for all samples was achieved for fields larger than $1 \mathrm{~T}$. XMCD spectra were obtained by reversing the polarization of the incoming circularly polarized light. The total electron yield detection technique was used due to its surface sensitivity. The escape depth of the secondary photoelectrons $(2-3 \mathrm{~nm})$ guarantees that the measured spectra are mainly determined by the $\mathrm{Mn}$ atoms close to the interfacial region.

\section{RESULTS}

LSMO has the highest ferromagnetic transition temperature $\left(T_{C} \sim 370 \mathrm{~K}\right)$ among the manganese perovskites with a strong magnetoresistive response and metallic conductivity below $T_{C}$. At low temperature, it is a half-metal ${ }^{22}$ and presents a rhombohedral $(R-3 c)$ crystal structure. In this work, we will use the pseudocubic notation in which the cell parameter $a$ is $a_{\text {bulk-LSMO }}=0.388 \mathrm{~nm}$. LCMO exhibits an orthorhombic (Pnma) crystal structure. In the pseudocubic notation, cell parameter $a$ is $a_{\text {bulk-LCMO }}=0.386 \mathrm{~nm}$. The ferromagnetic transition temperature of bulk LCMO is $T_{C} \sim 270 \mathrm{~K}$, and it also exhibits strong magnetoresistive response and metallic conductivity below $T_{C}$. However, it has a narrower conduction band rendering it then more inclined to exhibit phase segregation phenomena. However, film-thickness reduction and structural strain might severely modify magnetic and transport properties with respect to that of the bulk material. Reciprocal space maps of the (103) reflections of LSMO and LCMO films grown on STO (see Fig. 1) indicate that the in-plane lattice constant of both LSMO and LCMO films perfectly match that of the underlying substrate $\left(a_{\text {Film }}=a_{\text {Subst }} \sim 0.3905 \mathrm{~nm}\right)$. Thus, LSMO films grown on STO substrate are under biaxial tensile strain $(+0.41 \%)$ as well as LCMO films $(+1.2 \%)$ with a slightly reduced out-of-plane cell parameter $c$ in both cases. In Fig. 2(a) we show $\theta-2 \theta$ scans of the (004) peak of the LSMO and LCMO samples. A $c$ parameter of about $0.3870(3) \mathrm{nm}$ is deduced for LSMO and about $0.3848(3) \mathrm{nm}$ for LCMO. However, in this latter case, tiny variations of $c$ are detected, which would suggest slightly different relaxation states [Fig. 2(b)]. Both $\theta-2 \theta$ scans and reciprocal space maps suggest that LSMO and LCMO films with the different capping layers are very homogeneous and coherently strained
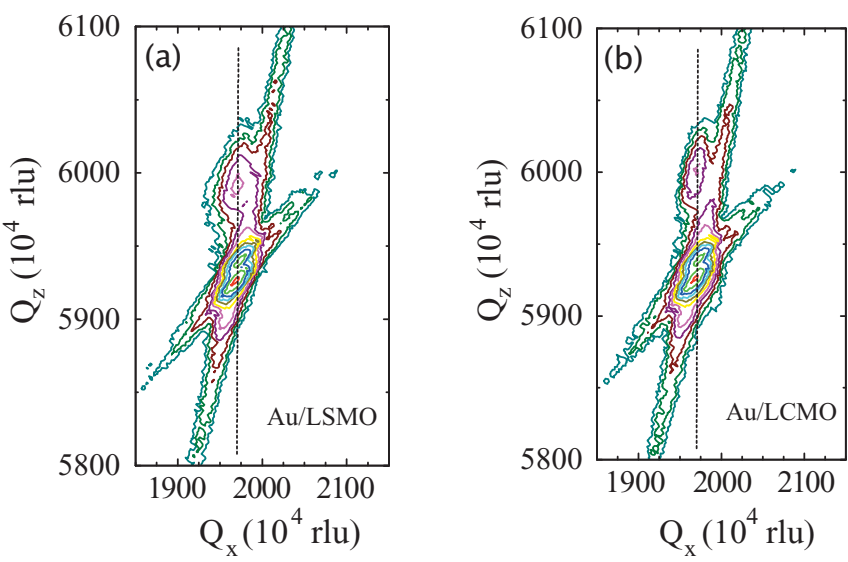

FIG. 1. (Color online) Reciprocal space diffraction map around the (103) reflection of (a) the LSMO/STO and (b) LCMO/STO films capped with an Au layer. 

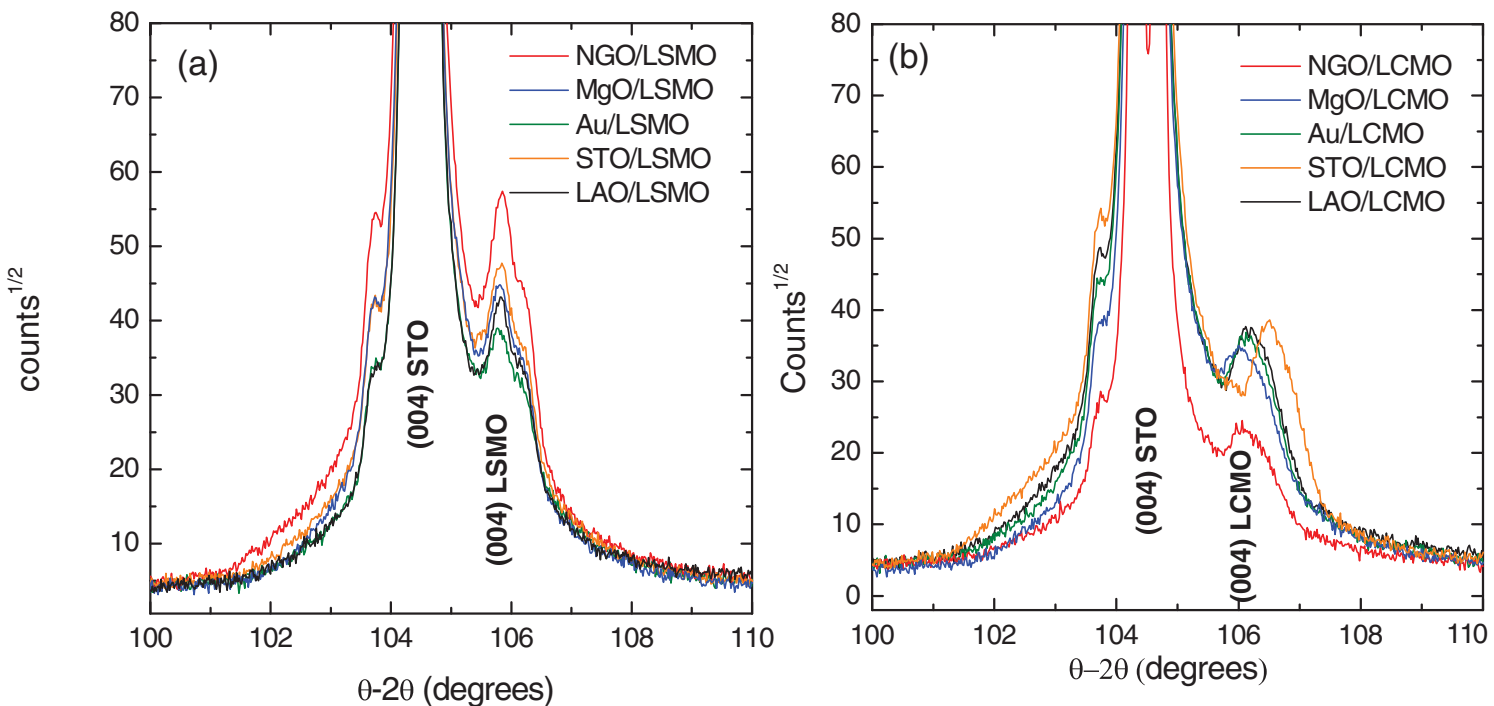

FIG. 2. (Color online) $\theta-2 \theta$ scans of the (004) peak of (a) LSMO and (b) LCMO samples prepared on top of (001)-oriented STO substrates. The different curves correspond to the different capping layers used.

in all the volume. On the other hand, the surface morphology of the samples after deposition of the capping layers is homogeneous and reproduces the underlying morphology of the manganite film in all the cases but for the Au capping (see Fig. 3). We will come later to this point.

In Fig. 4(a), we show the temperature dependence of the magnetization of the different LSMO samples measured in an applied magnetic field of $5 \mathrm{kOe}$. It is found that the ferromagnetic transition temperature $T_{C}$ is very similar in all the samples $\left(T_{C} \sim 350 \mathrm{~K}\right)$ and slightly below that of the bulk material $\left(T_{C} \sim 370 \mathrm{~K}\right)$. This slight reduction of $T_{C}$ can be explained in terms of oxygen deficiency and strain. The very same comments are also valid for LCMO samples [see Fig. 4(b)]; $T_{C}$ values are also very similar $\left(T_{C} \sim 250 \mathrm{~K}\right)$ and slightly below that of the bulk material $\left(T_{C} \sim 270 \mathrm{~K}\right)$. Thus, we can safely exclude severe effects of the capping on the robustness of double-exchange ferromagnetism in our samples, at least when considering the whole film volume. It is worth mentioning that in both LSMO and LCMO samples $\mathrm{MgO}$ capping causes an extra reduction of $T_{C}$ that deserves further study. Moreover, $M(T)$ curves in Fig. 4 show that the saturation magnetization $M_{S}$ is below that of the bulk value $\left(M_{S} \sim 590 \mathrm{emu} / \mathrm{cm}^{3}\right)$, and it seems to be correlated with the capping material. Nevertheless, we should clarify that this is only due to the fact that the samples are not magnetically saturated at the field used for the $M(T)$ measurements $(H=$ $5 \mathrm{kOe})$. When higher magnetic field values are applied, $M_{S}$ values close to that of the bulk material are found in all the cases, except for the samples capped with $\mathrm{MgO}$, which exhibits a reduction of $M_{S}$ of about $5 \%$.

The Mn XAS at the $L_{3,2}$-edge of LSMO (a) and LCMO (b) films with different cappings together with that of uncapped

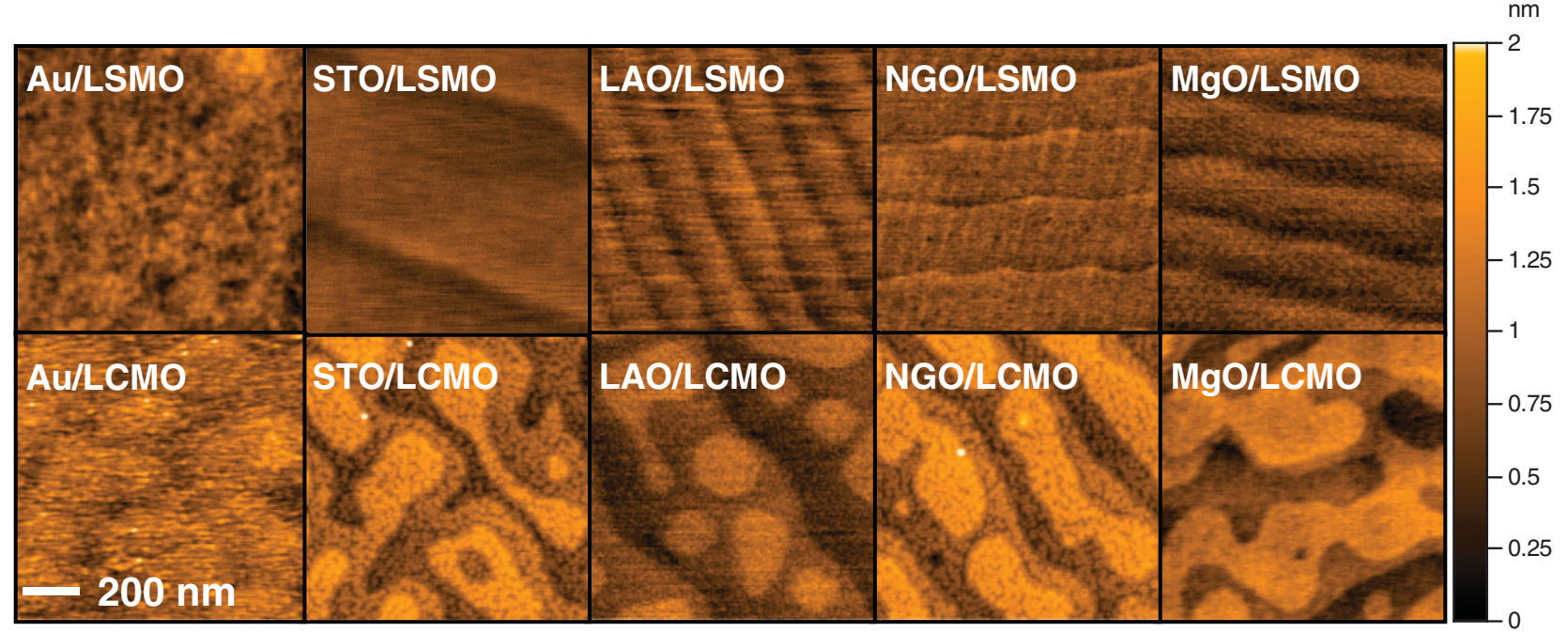

FIG. 3. (Color online) AFM topography of the different samples obtained in tapping mode. 

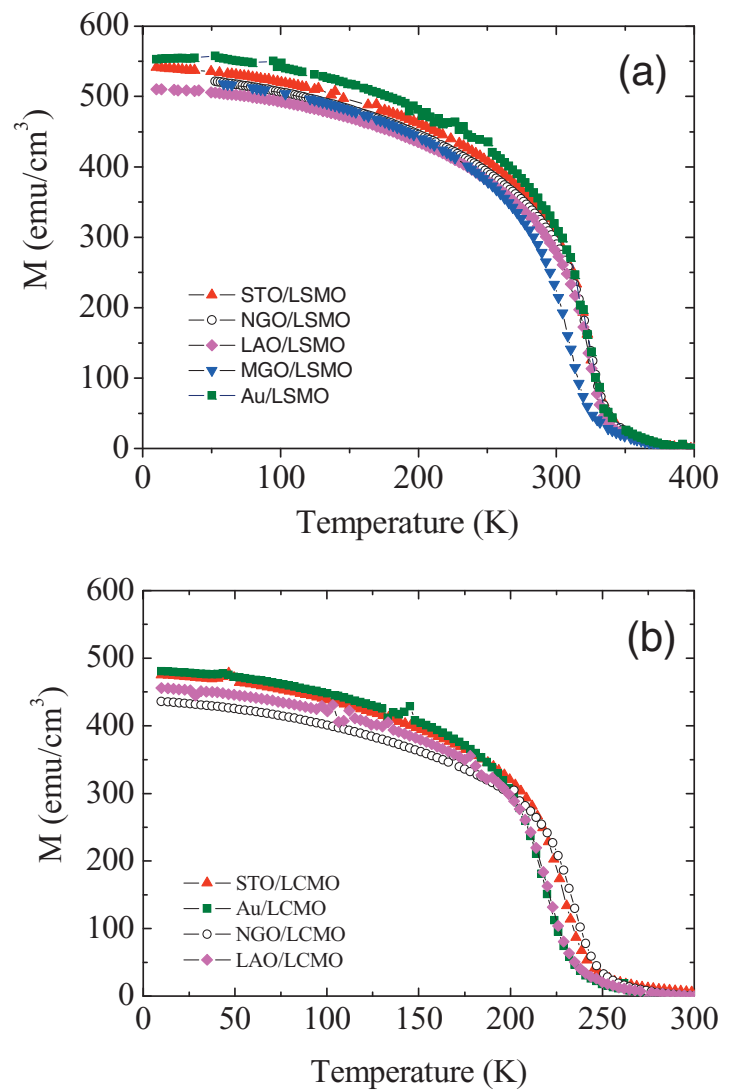

FIG. 4. (Color online) Magnetization versus temperature $M(T)$ curves for (a) LSMO and (b) LCMO samples with different capping layers in a magnetic field of $5 \mathrm{kOe}$.

LSMO and LCMO bulklike reference spectra are depicted in Fig. 5. The XAS have been measured at $T=10 \mathrm{~K}$ and $H=0$ after zero-field cooling. The XAS has been obtained by averaging the spectra measured with left and right incoming circularly polarized light.

Both LSMO and LCMO interfaces exhibit similar trends in the $\mathrm{Mn} L_{3,2}$-edge spectrum, and thus in the Mn oxidation state, as a function of the capping layer. Reference Mn $L_{3,2}$-edge spectra are like those reported for bulk LCMO and LSMO. ${ }^{19}$ From now on, we will consider these reference spectra as representative for the bulk LSMO and LCMO compounds. Samples with LAO capping exhibit almost bulklike spectra. Some minute differences when compared to the respective reference spectra are present at the low-energy side of the $L_{3}$ peak $(\sim 641 \mathrm{eV})$ in both cases. According to Abbate et al. ${ }^{23}$ this can be related to a tiny (below $1-2 \%$ ) reduction (increase) of the $\mathrm{Mn}^{4+}\left(\mathrm{Mn}^{3+}\right)$ content at the interface. In the case of NGO and $\mathrm{MgO}$ capping, the obtained curves are clearly different to those recorded for LAO capping. In both cases, the corresponding XAS spectra show increased intensity with respect to the reference spectrum at the high-energy side of the $L_{3}$ peak $(643-645 \mathrm{eV})$. Spectral shifts towards higher energy values are also observed, thus indicating an increase of the oxidation state of the $\mathrm{Mn}$ ions, i.e. an increase in the $\mathrm{Mn}^{4+}$ content at the interface. Comparison with the reference sample shows that the increase in the $\mathrm{Mn}^{4+}$ content at the interface amounts $\sim 15-18 \%$ in both $\mathrm{LSMO} / \mathrm{MgO}(\mathrm{NG} 0)$
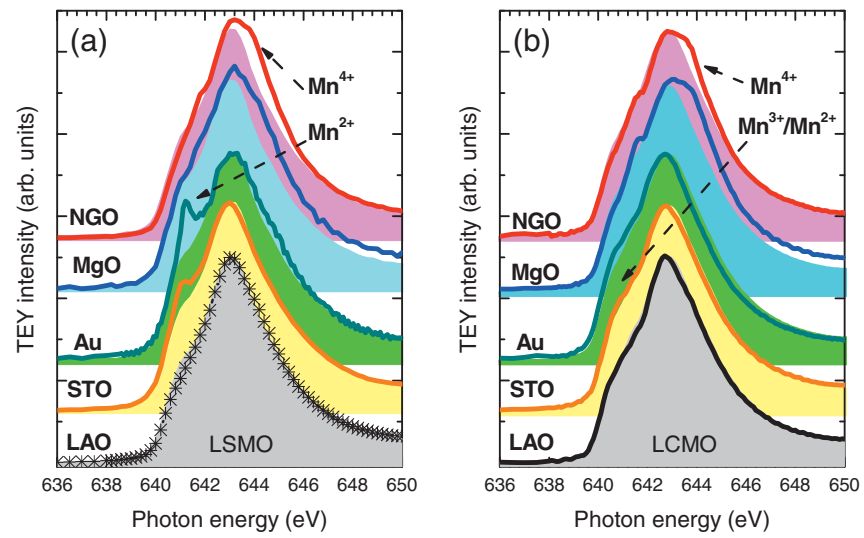

FIG. 5. (Color online) Mn $L_{3}$-edge absorption spectra for (a) LSMO and (b) LCMO films with different cappings obtained at $H=0 \mathrm{~T}$ after zero-field cooling (see text for details). Spectra are vertically shifted in order to allow better comparison. The reference spectra are shown by filled curves. Both STO and Au capping lead to a low-energy feature associated with $\mathrm{Mn}^{2+}$ formation. $\mathrm{MgO}$ and NGO capping promote the appearance of an excess of $\mathrm{Mn}^{4+}$ by the interface, whereas LAO capping does not produce a significant modification.

and $\mathrm{LCMO} / \mathrm{MgO}(\mathrm{NGO})$ interfaces. On the contrary, samples with STO and Au capping present an increase of intensity at the low-energy side of the $\mathrm{Mn} L_{3}$ peak $(641.2 \mathrm{eV})$. A concomitant energy shift of $0.1 \mathrm{eV}$ towards lower energies rules out $\mathrm{Mn}^{4+}$ as its origin. In addition, the size of the spectral feature at $641.2 \mathrm{eV}$ observed for the case of LSMO films also rules out $\mathrm{Mn}^{3+}$ (Ref. 23) and thus reveals the presence of divalent Mn. ${ }^{19}$ Following the procedure described in Ref. 19, the amount of $\mathrm{Mn}^{2+}$ at the LSMO/STO interface can be estimated to be about $6 \%$, while it is below $3 \%$ for LCMO. The appearance of $\mathrm{Mn}^{2+}$ at the interface is more pronounced in Au capped samples, especially in the LSMO/Au system where $\mathrm{Mn}^{2+}$ amounts to about $14 \%$ (in the case of LCMO, it is only about $4 \%$ ).
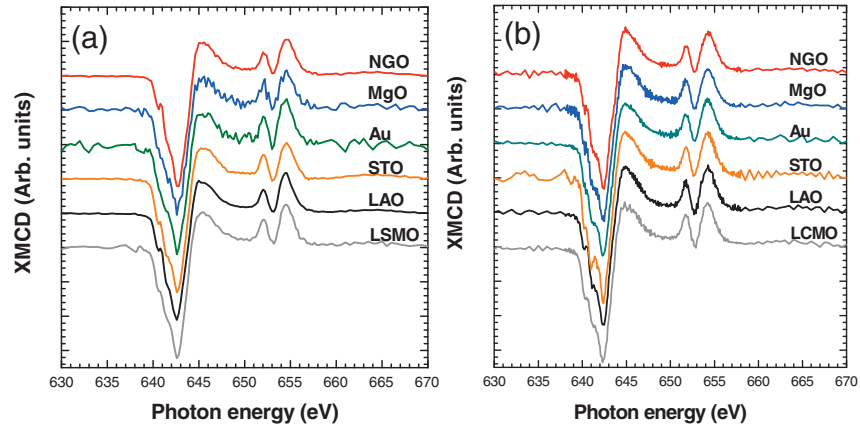

FIG. 6. (Color online) XMCD spectra corresponding to (a) LSMO and (b) LCMO films with different capping layers. Magnetic saturation is obtained for fields larger than $1 \mathrm{~T}$ in all cases. Depicted LSMO and LCMO XMCD curves have been obtained at $2 \mathrm{~T}$ and $3 \mathrm{~T}$, respectively. Spectra are vertically shifted in order to allow better comparison. All spectra look alike pointing to the presence of a single FM phase corresponding to the nominal composition. 


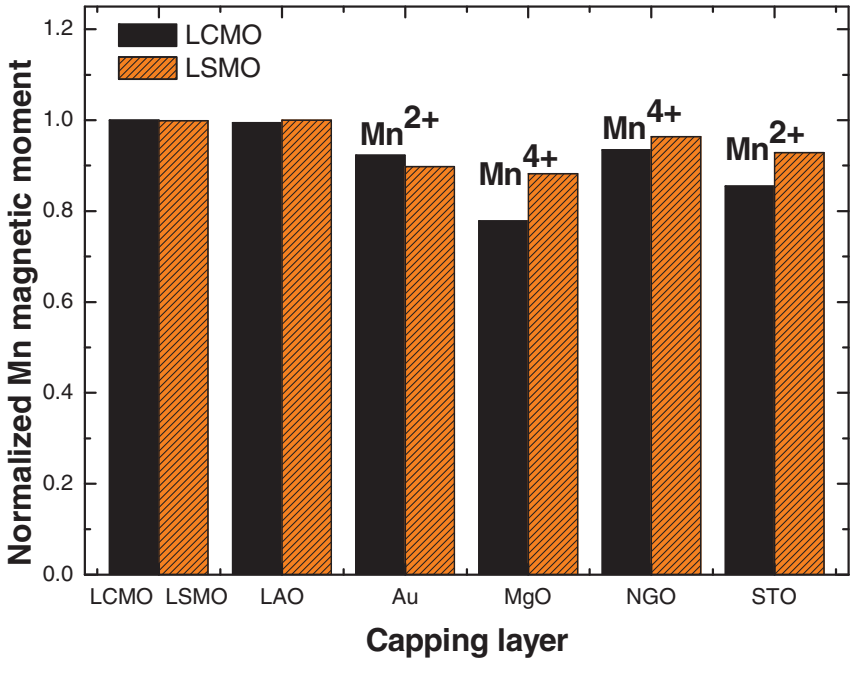

FIG. 7. (Color online) Interface magnetization for LSMO and LCMO thin films as a function of the capping layer used. Depicted data correspond to values obtained at $H=2 \mathrm{~T}$ in the case of LSMO and $H=3 \mathrm{~T}$ in the case of LCMO thin films, i.e. well above saturation $(H \approx 1 \mathrm{~T})$. Values have been obtained by using the sum rules and normalizing to the uncapped LSMO or LCMO layers (labeled as uncapped).A reduction of the magnetization is detected in those cases where capping also induces modification of the Mn oxidation state at the interface, that is, $\mathrm{Au}, \mathrm{STO}, \mathrm{MgO}$, and NGO.

The magnetic properties of the interface were studied by using XMCD. XMCD measures the dependence of the X-ray absorption spectrum of a ferromagnetically ordered sample on the helicity of circularly polarized incident radiation. Experimentally it is determined as the difference between two absorption spectra measured with opposite helicities $(\mu+$ and $\mu-$ ). Spectra corresponding to the different interfaces studied are shown in Fig. 6. The XMCD spectra of the samples are very much like the uncapped reference ones. This precludes the presence of any ferromagnetic phase besides that expected according to the nominal composition. By using the so-called sum rules, it is possible to quantitatively determine the spin and orbital contributions to the magnetic moment. ${ }^{24}$ However, this is not a trivial process, and in the case of $\mathrm{Mn}$, the sum rules yield values with an error larger than $50 \%$ for the spin moment. ${ }^{25}$ This prevents, in our case, an exact determination of the interface magnetization; however, a comparison between the different samples can be performed. In Fig. 7, we depict the magnetic moment $\left(m_{\text {spin }}+m_{\text {orb }}\right)$ per atom for the different interfaces of LCMO and LSMO films normalized to the value obtained for the corresponding uncapped (bulklike) samples. Our results indicate that all capping, except $\mathrm{LAO}$, lead to depressed magnetic moments at the interface in agreement with the departure from the $\mathrm{Mn}^{3+} / \mathrm{Mn}^{4+}$ valency balance corresponding to the $2 / 3-1 / 3$ nominal composition.

\section{DISCUSSION AND CONCLUSIONS}

Although strain effects are important at the substratemanganite interface, ${ }^{16}$ they should not play a major role in the manganite-insulating barrier interface. The insulating barrier is very thin, and the lattice matching should proceed by adapting the cell parameters of the material of the insulating barrier to that of the manganite. In high-quality heterostructures, epitaxial growth with flat atomically sharp interfaces has been achieved ${ }^{3,9,10,16}$ with a very low occurrence of structural defects. This is so because, in most of the cases, the thicknesses of the insulating barrier overlayers are smaller than the critical thickness at which a first relaxing defect appears. ${ }^{26}$ Thus, in principle, strain and structural defects should not represent a relevant task at force in modifying the magnetotransport properties of the manganite-insulating barrier interface. Therefore, in high-quality epitaxial heterostructures, the degradation of the magnetotransport properties at the manganite-insulating barrier interface would be mainly due to the variations of the oxygen stoichiometry and/or doping level.

To gain access to the electronic and magnetic structure at the interface, surface-sensitive probes are required. The magnetic properties of the surface and interfaces in manganites have been studied by using different techniques. Surfacesensitive photoemission spectroscopy studies of $\mathrm{LSMO}^{27}$ indicate a degraded average magnetization in the surface region. Nonlinear magneto-optical effects can also be used to probe interface magnetism ${ }^{28}$ and in fact have been used to test STO/LSMO/LAO superlattices, ${ }^{8}$ showing that STO suppresses ferromagnetic spin ordering at the interface more significantly than LAO does. Magnetization-induced secondharmonic generation has also been used to study magnetic properties at LSMO/STO and LSMO/LAO interfaces, ${ }^{3}$ yielding similar conclusions, i.e. STO induces antiferromagnetic spin canting at the interface, while ferromagnetic alignment is less affected by LAO. These observations have been explained by considering that interfacial LSMO behaves as an $A$-type antiferromagnet due to a possible hole doping from STO. ${ }^{8,29}$ These conclusions have been obtained within a double-exchange scenario, where only mixed valence $\mathrm{Mn}^{3+/ 4+}$ or separate $\mathrm{Mn}^{3+}$ and $\mathrm{Mn}^{4+}$ oxidation states are taken into account. This fact, correct when considering the oxidation state of the parent compounds $\left[\mathrm{LaMnO}_{3}\right.$ and $\left.\mathrm{Sr}(\mathrm{Ca}) \mathrm{MnO}_{3}\right]$, might not always be supported on an experimental basis since the oxidation state of the $\mathrm{Mn}$ atoms are rarely probed. When done, ${ }^{9,10,17}$ divalent Mn has not been considered based again on stoichiometry considerations. Our results reveal, however, a completely different picture. As shown in Fig. 5, STO-capped LSMO and LCMO films present increased TEY (absorption) intensity at $\sim 641.2 \mathrm{eV}$. Such a spectral feature is usually assigned to the presence of $\mathrm{Mn}^{4+}$; however, its high intensity (especially in the LSMO case) as well as the shift towards lower energies of the spectrum as compared to the reference samples reveals the presence of $\mathrm{Mn}^{2+}$. Indeed the presence of $\mathrm{Mn}^{2+}$ ions by the interface indicates a strong disruption of the $\mathrm{Mn}^{3+} / \mathrm{Mn}^{4+}$ valency balance and would even explain the observation of a magnetically dead layer. ${ }^{19}$ This scenario thus suggests an electron doping of LSMO at the interface with STO in agreement with the results reported in Ref. 9, the main difference being, however, the formation of divalent $\mathrm{Mn}$ instead of the increase of the $\mathrm{Mn}^{3+} / \mathrm{Mn}^{4+}$ ratio. The discrepancy between the electronand hole-doped schemes is usually explained in terms of the stacking sequence at the interface: $\mathrm{La}_{2 / 3} \mathrm{Sr}_{1 / 3} \mathrm{O} / \mathrm{TiO}_{2}$ (type I), for which electrostatic equilibrium imposes the pres- 
ence of extra electrons at the interface, or $\mathrm{MnO}_{2} / \mathrm{SrO}$ (type II), which generates hole doping of the $\mathrm{MnO}_{2}$ layer. Interestingly, assuming the same stacking sequence for LSMO/LAO (samples have been prepared following the very same procedure and in the same evaporation system), the stacking at the interface should be $\mathrm{La}_{2 / 3} \mathrm{Sr}_{1 / 3} \mathrm{O} / \mathrm{AlO}_{2}$, for which electrostatic equilibrium would impose extra holes at the interface that seem to be much less detrimental for the magnetotransport properties. Nevertheless, in our samples, LAO capping has almost no detectable effect on the Mn oxidation state or on the magnetic properties in close agreement with Yamada et al. ${ }^{3}$

As mentioned above, the formation of $\mathrm{Mn}^{2+}$ by the interface is more evident in Au-capped samples, especially in the LSMO/Au system, which should cause a severe degradation of the magnetic and magnetotransport properties at the interface region. In fact, Brivio et al. ${ }^{30}$ have recently reported a dramatic degradation of the ferromagnetic features in very thin LSMO layers after Au capping. This strong degradation disappears for LSMO thickness above $8 \mathrm{~nm}$, thus confirming that it is an interfacial effect. Brivio et al. attribute the observed effects to a notable reduction of the Mn oxidation state motivated by an inhomogeneous oxygen dragging from the manganite due to the high reactivity of Au nanoparticles that form on top of the LSMO layer for thin $\mathrm{Au}(\sim 2 \mathrm{~nm})$ capping layers. These results are in very good agreement with our data that also indicate a reduction of the Mn oxidation state at the interface. It is worth mentioning that, in LCMO samples, the effect is much less important and would reflect differences in Au wetability on top of LSMO and LCMO layers, precluding the formation of $\mathrm{Au}$ nanoparticles in the latter, reducing the oxygen dragging effect. Interestingly enough, Au capping is the only case in which AFM pictures do not show coherence of the capping layer with the underlying manganite film (see Fig 3). This is more evident in the case of the LSMO/Au sample, thus suggesting that the mechanism proposed in Ref. 30 would also apply in our samples. Also, $\mathrm{Mn}^{2+}$ formation due to direct contact between LSMO or LCMO interface with ambient air due to inhomogeneous $\mathrm{Au}$ capping cannot be excluded; ${ }^{19}$ although, in such a case, we would also expect divalent Mn presence on the spectra corresponding to the reference samples.

In the case of the other two capping layers studied, namely $\mathrm{MgO}$ and $\mathrm{NGO}$, the XAS data reveal a significant increase of their $\mathrm{Mn}^{4+}$ content. A mechanism to explain this increase could be, for instance, the formation of a thin layer of a different oxide due to dilution of some $\mathrm{Nd}, \mathrm{Ga}$, or $\mathrm{Mg}$ atoms. In the latter case, for example, $\mathrm{MgMnO}_{3}$ could be formed at the interface. ${ }^{31}$ The formation of such a thin layer would drastically distort the magnetotransport properties of the interface.

The normalized magnetic moments per atom depicted in Fig. 7 indicate that all capping, except LAO, lead to depressed magnetic moments at the interface in agreement with the above-described XAS observations of deviations from the nominal $\mathrm{Mn}^{3+/ 4+}$ mixed-valence state. This magnetization reduction could be related to spin canting of interfacial $\mathrm{Mn}$ moments, as reported, for instance, in Refs. 3 and 29, or to a modification of the nominal $\mathrm{Mn}^{3+} / \mathrm{Mn}^{4+}$ ratio. Without excluding such a scenario, our data reveal the presence of another phase. The XMCD spectra depicted in Fig. 6 look like that of the bulklike reference sample for all films independently of the capping, thus indicating the presence of the expected ferromagnetic phase and excluding the presence of any other FM phase besides that according to the nominal composition. It is important to remark that the sum rules calculate the magnetic moment per atom. ${ }^{24}$ In order to obtain both $m_{\text {spin }}$ and $m_{\text {orb }}$, it is necessary to calculate the integral of the XAS spectrum $\left(I_{\text {XAS }}\right)$. Such $I_{\text {XAS }}$ should only arise from the XAS spectrum corresponding to the FM atoms. Any nonmagnetic contribution would lead to an overestimation of $I_{\text {XAS }}$ and thus to an underestimation of $m_{\text {tot }}{ }^{24}$ This is indeed the present case. Since the XMCD reveals no other FM phase than that expected, we concluded that both $\mathrm{Mn}^{2+}$ and $\mathrm{Mn}^{4+}$ excess are not FM. Our data thus suggest a phase separation scenario at the film-capping interface. At least two phases coexist; one FM phase with nominal composition $(x=1 / 3)$ and a second one nonferromagnetic with compositions, reflecting the excess of $\mathrm{Mn}^{2+}$ or $\mathrm{Mn}^{4+}$ in the case of Au and STO and in the case of $\mathrm{MgO}$ and $\mathrm{NGO}$, respectively. Since the observed changes appear only due to the different capping (no strain involved) the data suggest an in-depth phase separation scenario, i.e. excess of $\mathrm{Mn}^{2+}$ or $\mathrm{Mn}^{4+}$ at the interface, leading to new nonmagnetic and most probably insulator phases on top of the bulklike LSMO or LCMO thin films. Finally, we would like to note that due to the luck of sensitivity of XMCD to antiferromagnetic ordering, we cannot exclude the presence of such a phase, which would certainly induce canting at the interface, as previously reported. ${ }^{3,29}$

In conclusion, we have analyzed the effect of different capping layers on the magnetic properties of LSMO and LCMO manganite thin films on top of (001)-STO substrates. Irrespective to the capping layer used, samples exhibit both $T_{C}$ and saturation magnetization close to that of the corresponding bulk material. This is so because the volume of material implicated in the interface is very small compared to the whole volume of the sample. However, we should mention that an abnormal reduction of about $5 \%$ in the value of $M_{S}$ is observed in the case of the $\mathrm{MgO}$ capping layer, which deserves further studies. The use of surface sensitive techniques, such as XAS and XMCD detected by means of TEY, allows direct access to study chemical composition and magnetic properties at the interfacial region. Our XAS data demonstrate that both LSMO and LCMO samples are locally modified at the interface in the case of $\mathrm{MgO}$ and $\mathrm{NGO}$ capping, showing a $\mathrm{Mn}^{4+}$ excess. Also, $\mathrm{Au}$ and $\mathrm{Mn}^{2+}$ lead to divalent $\mathrm{Mn}$ formation. In contrast, LAO capping does not seem to disturb the interface. In concomitance with those results, XMCD data make evident a degradation of the magnetic properties on a per-atom basis in those cases where Mn oxidation state departs from the nominal $\mathrm{Mn}^{3+} / \mathrm{Mn}^{4+}$ valency balance. Our data does not agree with spin canting at the interface, reducing the magnetization, but to a phase separation scenario where at least two phases, one FM and another non-FM, coexist at the interface.

\section{ACKNOWLEDGMENTS}

We acknowledge financial support from the Spanish MEC (MAT2009-08024), CONSOLIDER (CSD2007-00041), and FEDER program. The research leading to these re- 
sults has received funding from the European Community's Seventh Framework Program (FP7/2007-2013) under Grant
No. 226716. Z.K. thanks the Spanish MEC for the financial support through the RyC program.
${ }^{1}$ A. Ohtomo and H. Hwang, Nature (London) 427, 423 (2004).

${ }^{2}$ M. Huijben, G. Rijnders, D. H. A. Blank, S. Bals, S. Van Aert, J. Verbeeck, G. Van Tendeloo, A. Brinkman, and H. Hilgenkamp, Nat. Mater. 5, 556 (2006).

${ }^{3}$ H. Yamada, Y. Ogawa, Y. Ishii, H. Sato, M. Kawasaki, H. Akoh, and Y. Tokura, Science 305, 646 (2004).

${ }^{4}$ S. Thiel, G. Hammerl, A. Schmehl, C. W. Schneider, and J. Mannhart, Science 313, 1942 (2006).

${ }^{5}$ J. Garcia-Barriocanal, J. C. Cezar, F. Y. Bruno, P. Thakur, N. B. Brookes, C. Utfeld, A. Rivera-Calzada, S. R. Giblin, J. W. Taylor, J. A. Duffy, S. B. Dugdale, T. Nakamura, K. Kodama, C. Leon, S. Okamoto, and J. Santamaria, Nature Communications 1, 1 (2010).

${ }^{6}$ E. Dagotto, T. Hotta, and A. Moreo, Phys. Rep. 344, 1 (2001).

${ }^{7}$ P. Schiffer, A. P. Ramirez, W. Bao, and S. W. Cheong, Phys. Rev. Lett. 75, 3336 (1995).

${ }^{8}$ H. Yamada, M. Kawasaki, Y. Ogawa, and Y. Tokura, Appl. Phys. Lett. 81, 4793 (2002).

${ }^{9}$ J. L. Maurice, D. Imhoff, J. P. Contour, and C. Colliex, Philos. Mag. 86, 2127 (2006).

${ }^{10}$ T. Riedl, T. Gemming, K. Dörr, M. Luysberg, and K. Wetzig, Microsc., Microanal. 15, 213 (2009).

${ }^{11}$ V. Garcia, M. Bibes, J. L. Maurice, E. Jacquet, K. Bouzehouane, J. P. Contour and A. Barthélémy, Appl. Phys. Lett. 87, 212501 (2005).

${ }^{12}$ C. Martinez Boubeta, Z. Konstantinovic, Ll. Balcells, S. Estradé, J. Arbiol, A. Cebollada, and B. Martínez, J. Cryst. Growth and Design 10, 1017 (2010).

${ }^{13}$ M. Esseling, V. Moshnyaga, K. Samwer, A. Marx, and R. Gross, Appl. Phys. Lett. 87, 082509 (2005).

${ }^{14}$ M. Bibes, S. Valencia, L1. Balcells, B. Martínez, J. Fontcuberta, M. Wojcik, S. Nadolski, and E. Jedryka, Phys. Rev. B 66, 134416 (2002).

${ }^{15}$ M. Angeloni, G. Balestrino, N. G. Boggio, P. G. Medaglia, P. Orgiani, and A. Tebano, J. Appl. Phys. 96, 6387 (2004).

${ }^{16}$ Ll. Abad, V. Laukhin, S. Valencia, A. Gaup, W. Gudat, L1. Balcells, and B. Martínez, Adv. Funct. Mater. 17, 3918 (2007).
${ }^{17}$ L. Samet, D. Imhoff, J. L. Maurice, J. P. Contour, A. Gloter, T. Manoubi, A. Fert, and C. Colliex, Eur. Phys. J. B 34, 179 (2003).

${ }^{18}$ C. Aruta, G. Ghiringhelli, V. Bisogni, L. Braicovich, N. B. Brookes, A. Tebano, and G. Balestrino, Phys. Rev. B 80, 014431 (2009).

${ }^{19}$ S. Valencia, A. Gaupp, W. Gudat, L1. Abad, Ll. Balcells, A. Cavallaro, B. Martinez, and F. J. Palomares, Phys. Rev. B 73, 104402 (2006); S. Valencia, A. Gaupp, W. Gudat, Ll. Abad, Ll. Balcells, and B. Martinez, ibid. 75, 184431 (2007).

${ }^{20}$ S. Valencia, Z. Kontantinovic, A. Gaupp, D. Schmitz, Ll. Balcells, and B. Martínez, J. Appl. Phys. 109, 07D718 (2011).

${ }^{21}$ Z. Konstantinovic, J. Santiso, D. Colson, A. Forget, Ll. Balcells, and B. Martinez, J. Appl. Phys. 105, 5 (2009).

${ }^{22}$ M. Bowen, M. Bibes, A. Barthélémy, J. P. Contour, A. Anane, Y. Lemaître, and A. Fert, Appl. Phys. Lett. 82, 233 (2003).

${ }^{23}$ M. Abbate, F. M. F. de Groot, J. C. Fuggle, A. Fujimori, O. Strebel, F. Lopez, M. Domke, G. Kaindl, G. A. Sawatzky, M. Takano, Y. Takeda, H. Eisaki, and S. Uchida, Phys. Rev. B 46, 4511 (1992).

${ }^{24}$ B. T. Thole, P. Carra, F. Sette, and G. van der Laan, Phys. Rev. Lett. 68, 1943 (1992); P. Carra, B. T. Thole, M. Altarelli, and X. D. Wang, 70, 694 (1993).

${ }^{25}$ C. Piamonteze, P. Miedema, and F. M. F. de Groot, Phys. Rev. B 80, 184410 (2009) and references therein.

${ }^{26}$ J. P. Contour, A. Abert, and A. Défossez, SPIE 2697, 339 (1996).

${ }^{27}$ J. H. Park, E. Vescovo, H. J. Kim, C. Kwon, R. Ramesh, and T. Venkatesan, Phys. Rev. Lett. 81, 1953 (1998).

${ }^{28}$ J. Reif, J.C. Zink, C. M. Schneider, and J. Kirschner, Phys. Rev. Lett. 67, 2878 (1991).

${ }^{29}$ M. Izumi, Y. Ogimoto, Y. Okimoto, T. Manako, P. Ahmet, K. Nakajima, T. Chikyow, M. Kawasaki, and Y. Tokura, Phys. Rev. B 64, 064429 (2001).

${ }^{30}$ S. Brivio, C. Magen, A.A. Sidorenko, D. Petti, M. Cantoni, M. Finazzi, F. Ciccacci, R. De Renzi, M. Varela, S. Picozzi, and R. Bertacco, Phys. Rev. B 81, 094410 (2010).

${ }^{31}$ B. L. Chamberland, A. W. Sleight, and J. F. Weiher, J. Solid State Chem. 1512 (1970). 